\title{
Current pharmacotherapy in the newborn
}

\author{
This article was published in the following Dove Press journal: \\ Research and Reports in Neonatology \\ II September 2012 \\ Number of times this article has been viewed
}

\section{Roberto Antonucci Annalisa Porcella \\ Division of Neonatology and Pediatrics, Department of Medicine, "Nostra Signora di Bonaria" Hospital, San Gavino Monreale, Italy}

Correspondence: Roberto Antonucci

Chief, Division of Neonatology and Pediatrics, "Nostra Signora di Bonaria" Hospital, San Gavino Monreale (VS), Italy Tel +390709378263

$\mathrm{Fax}+390709378260$

Email roant@tiscali.it
Abstract: Several drugs are used in newborns in spite of the lack of specific clinical research in this particularly vulnerable population with particular needs. In the newborn, the individual response to a drug in terms of efficacy and safety is highly variable, and predicting drug dosing is complex since rapid physiological changes occurring during the perinatal and early postnatal periods affect the pharmacokinetic profile of many drugs. Neonatal disorders such as renal and hepatic diseases may also have significant implications for drug pharmacokinetics. Therefore, pharmacotherapy in the newborn brings difficulties in accurate drug delivery and carries a high risk of adverse drug reactions. In addition, the neonatal population, especially that treated in neonatal intensive care units, is highly exposed to the risk of medication errors, with potentially serious adverse events. This paper reviews some current issues related to neonatal pharmacotherapy that are of paramount importance for the clinician. In particular, the peculiar pharmacokinetics of drugs during the neonatal period and its clinical implications are discussed. The use of therapeutic drug monitoring to individualize drug dosage and to optimize pharmacotherapy is also described. Finally, the relevant issue of medication errors in neonatology is examined in order to highlight their main causes and key strategies in preventing these type of errors. In the future, pharmacometabolomics and other "omic" sciences could play an important role in designing personalized neonatal health care.

Keywords: pharmacotherapy, drugs, pharmacokinetics, therapeutic drug monitoring, medication errors, newborn

\section{Introduction}

Since 1968, children have been systematically excluded from studies conducted to establish the efficacy and safety of new drugs. Ethical and medicolegal issues, logistic and technical difficulties, and economics of drug development are the main barriers to pharmaceutical research in neonates and children. At present, there is no pediatric safety or prescribing information in about $80 \%$ of drugs approved and labeled for adults. Many drugs that are given to neonates and children are "unlicensed," ie, they are not yet registered by the regulating authority that can ensure their safety, efficacy, and quality. On the other hand, other drugs, which are referred to as "off-label", are prescribed to children outside the terms of the product license with particular reference to indication, age, formulation, route of administration, or dosage. The "off-label" medication use may expose infants to the risk of adverse effects due to under- or overdosing, or of other effects not predictable from experience in adults. ${ }^{1}$

Drug therapy is strongly influenced by age: from a pharmacological viewpoint, neonates are not just "small children" and children are not just "small adults." In fact, 
the newborn can be considered as an extremely dynamic organism in which the absorption, distribution, metabolism, and excretion systems are rapidly developing. ${ }^{2}$ Consequently, the individual response to a drug in terms of efficacy and toxicity is highly variable in this group of patients, and predicting drug doses for them is complex. ${ }^{3}$ Constitutional, environmental, and genetic factors were found to influence phenotypic variation in drug metabolism during neonatal life. ${ }^{4}$ Clinical pharmacology is an area of study that is concerned with the integration of the pharmacokinetic and pharmacodynamic profile of a drug in order to optimize drug therapy. Drugs administered using pharmacokinetic and pharmacodynamic dosing approaches exhibit better response rates and less toxicity. ${ }^{3}$ Pharmacokinetics is the quantitative study of the absorption, distribution, metabolism, and excretion of drugs. The evaluation of the pharmacokinetic parameters of a drug allows an accurate determination of the dose and dose interval in order to achieve a defined target concentration, a desired pharmacological effect, or both. On the other hand, pharmacodynamics quantifies the response of the target body tissues to a measured concentration of a drug. The response to pharmacological agents may be desirable (effectiveness) or untoward (toxicity).

A number of amplified or deleterious drug effects reported in pediatric populations are related to differences in receptor sensitivity, but especially in drug disposition. These effects are generally more dramatic in neonates, since their response to a pharmacological treatment can be affected by various perinatal and postnatal conditions. The differential number and maturity of receptors may explain some of the differences in dose requirements in newborns. For example, newborns exhibit particular sensitivity to nondepolarizing neuromuscular agents (pancuronium, vecuronium) while they are resistant to depolarizing neuromuscular agents (succinylcholine). On the other hand, differences in absorption, distribution, excretion and, more frequently, in metabolism can modify drug disposition.

In this review, the multifaceted, rapidly evolving field of neonatal pharmacotherapy has been discussed, with a special focus on three topics that are particularly important in clinical settings: drug pharmacokinetics, therapeutic drug monitoring, and medication errors. An in-depth knowledge of these topics on the part of health care providers constitutes the basis for optimizing drug use and reducing adverse drug reactions.

\section{Pharmacokinetics in the neonate}

Once a drug has been administered, it will then undergo four processes: absorption, distribution, metabolism, and excretion.
The drug composition, the dose of drug, the patient's condition, and other therapeutic and environmental issues may affect the effectiveness of these processes.

In the newborn, physiological variables such as gestational and postnatal age strongly influence the maturational and developmental processes of various organs, which in turn affect drug pharmacokinetics. In addition, neonatal diseases secondary to prematurity or other causative factors are able to modify drug pharmacokinetics, as discussed in the following sections.

\section{Absorption}

To be effective, a drug must be absorbed from its site of administration into the systemic circulation, and subsequently it must be distributed to its site of action.

\section{Gastrointestinal absorption}

Drugs administered orally must cross the gastrointestinal barrier before entering the systemic circulation and being transported to their site of action. Most drugs are absorbed from the gastrointestinal tract by a mechanism of passive diffusion of un-ionized molecules through lipophilic membranes.

The rate and extent of drug absorption is influenced by some age-dependent patient variables, especially gastric $\mathrm{pH}$, gastric emptying time and biliary function. The greatest changes of $\mathrm{pH}$ and transit time of the gastrointestinal tract occur during the first month of life. ${ }^{5}$

In the early hours after birth, the gastric $\mathrm{pH}$ is neutral ( $\mathrm{pH}$ 6-8) due to the presence of ingested amniotic fluid in the stomach. ${ }^{6,7}$ Within the first 48 hours of life, gastric $\mathrm{pH}$ starts to fall due to the gastric secretion of hydrochloric acid and reaches a value of $1-3$ at about 6 months of life. ${ }^{6,7}$ However, in premature infants, gastric $\mathrm{pH}$ remains high for longer periods because of the reduced acid secretion. The $\mathrm{pH}$ at the site of absorption influences the extent of absorption. In a low $\mathrm{pH}$ environment, acidic drugs with a low $\mathrm{pK}$ are more un-ionized and more able to cross lipid membranes. ${ }^{1}$ This mechanism can explain the greater absorption of acid-labile drugs (eg, penicillin and ampicillin) compared to other drugs such as phenobarbital and phenytoin.

Another factor affecting drug absorption is gastric emptying: if it is slow, the drug is delayed in reaching the small intestine, where it is absorbed into the bloodstream. Gastric emptying is prolonged in the infant and in the neonate, particularly in the preterm newborn, and is influenced by type of feed (human milk and low-calorie feed increase the speed of gastric emptying) and by disease states (eg, pyloric stenosis and congestive cardiac failure). ${ }^{1}$ It reaches 
adult rates ( 10 to 20 minutes) at approximately 6 to 8 months of age. Therefore, drug absorption appears to be reduced and delayed after birth, with gradual improvement during the first 3 months.

As regards biliary function, this develops over the first month of life. In particular, both bile acid salts and pancreatic enzymes are reduced in the newborn, thus affecting the absorption of some lipophilic medications.

\section{Intramuscular, dermal, and rectal absorption}

Intramuscular absorption in the neonate and infant is variable and unpredictable because of decreased muscle tone, and variable perfusion and oxygenation. ${ }^{6}$ This route of administration is generally avoided in the neonatal period.

The dermal absorption of a compound is directly related to skin hydration and inversely related to the thickness of the stratum corneum. Compared with adult skin, the neonatal and infant skin, which exhibits an underdeveloped stratum corneum and an increased hydration, is more permeable to topically applied drugs. In addition, the ratio of the skin surface area to body weight is approximately three times greater in the newborn than in the adult. These characteristics of skin make topical drug formulations an important means of drug delivery in the newborn and infant. Well-documented pediatric cases of neurotoxicity and death have been described after accidental percutaneous hexachlorophane absorption. ${ }^{8}$

Rectal administration conduces to variable plasma concentrations, being influenced by lower gastrointestinal motility and depth of drug insertion. In fact, unlike the absorption via the upper rectal veins, the absorption via the inferior and middle rectal veins bypasses the hepatic firstpass metabolism. ${ }^{1}$

\section{Distribution}

The tissue distribution and the volume distribution (Vd) of drugs may be affected by a number of factors. ${ }^{9}$ Body water content declines and body fat increases with age; accordingly, newborns and young children have a lower body fat content and higher total body water when compared to adults. The greatest changes in body water compartments occur during the first year of life. Age-related changes in body composition influence the distribution of both lipidand water-soluble drugs. For example, lipid-soluble drugs such as diazepam have lower Vds in neonates and infants compared with adults. On the contrary, water-soluble drugs such as atracurium need to be administered at a higher dose per kilogram in the newborn to achieve adequate plasma and tissue concentrations. ${ }^{1}$
Table I Factors influencing drug distribution in the newborn.

- High total body water content (high extracellular fluid volume)

- Low body fat content

- High membrane permeability (central nervous system)

- Low levels of plasma drug-binding proteins (albumin, alpha I-acid glycoprotein)

- Low albumin affinity for some drugs

An increased membrane permeability allowing easier drug diffusion into compartments such as the central nervous system (CNS) is observed especially in preterm neonates but also in term neonates, particularly those with sepsis, hypoxia, and acidosis. This feature, combined with increased receptor sensitivity to many drugs, makes neonates more susceptible to the effects of centrally acting pharmacological agents.

Another important component in determining $\mathrm{Vd}$ is plasma protein binding. In fact, the extent to which a drug is bound to plasma proteins directly affects the distribution characteristics of the drug. Only the unbound drug can be distributed from the vascular bed into other biofluids and tissues, where it binds to its receptor and evokes a response. The most important drug-binding proteins in serum, which are albumin and alpha 1-acid glycoprotein, are decreased in the neonate. In addition, neonates exhibit a decreased affinity of albumin for drugs such as phenobarbital, phenytoin, and theophylline. Competitive binding by free fatty acids and bilirubin, which are present in higher concentrations in neonates, also contributes to the reduced albumin affinity observed for some drugs. The overall effect is a larger free fraction of drug in serum, and consequently an increased effect of the drug. The major factors influencing drug distribution in the newborn are summarized in Table 1.

\section{Metabolism}

Drug metabolism generally transforms a lipophilic parent compound into a more polar, water-soluble metabolite, thus facilitating drug elimination into the urine or bile. Other metabolic effects include the generation of an active metabolite or prodrug activation.

The major organ for drug metabolism is the liver. Hepatic blood flow and enzyme maturation mainly affect the ability of the newborn to metabolize drugs. ${ }^{1}$ The rate of the cardiac output delivered to the liver increases during newborn maturation. ${ }^{4}$ On the other hand, a developmental variation in the activity of drug-metabolizing enzymes has been attributed to changes in liver size and in the intrinsic activity of hepatic enzymes. ${ }^{10}$ 
Drug metabolism within the hepatocyte involves two major reactions, termed Phase I and Phase II reactions. Phase I, or nonsynthetic, reactions add a functional group to the parent molecule via oxidation, reduction, methylation, or hydroxylation. The majority of Phase I reactions are catalyzed by a superfamily of enzymes referred to as cytochrome P450 (CYPs). In contrast, Phase II, or synthetic reactions (conjugation) entail the attachment of a water-soluble molecule to the drug via glucuronidation, sulfation, or acetylation. In other words, Phase I reactions introduce or reveal a functional group within the drug molecule that serves as a site for a Phase II conjugation reaction; the conjugation with endogenous substrates such as acetate, glucuronic acid, glutathione, glycine, and sulfate (Phase II reactions) further increases the polarity of an intermediate metabolite, thus enhancing its renal excretion.

Both Phase I and Phase II metabolic pathways, which are immature at birth, are subject to maturational changes. It should also be considered that drug exposure during pregnancy may alter the postnatal metabolism. The infants prenatally exposed to phenobarbital exhibit a more rapid metabolism of this drug, due to a mechanism of enzyme induction. ${ }^{7}$

The CYP450 enzyme system is a supergene family, with 16 or more primary enzymes and a number of isozymes of specific gene families. The human CYP isozymes most responsible for drug metabolism are CYP1A2, CYP2C9, CYP2C19, CYP2D6, CYP2E1, and CYP3A4. Although the CYPs are found mainly in the liver, significant CYP expression and activity are also present in gastrointestinal mucosa, kidney, brain, and lung. ${ }^{11}$

The activity of hepatic drug-oxidizing enzymes is reduced during neonatal age. ${ }^{3,6}$ Therefore, body elimination for drugs depending on oxidation pathways, such as phenytoin, diazepam or caffeine is prolonged. ${ }^{3}$ The hepatic cytochrome $\mathrm{P} 450$ monooxygenase enzymes rapidly mature after birth, attaining adult metabolic activity at about 6-12 months of age. ${ }^{3,6}$

Clinicians should know the substrates for specific isozymes and the effects that particular drugs may have on isoenzyme activity (induction, inhibition) in order to predict the occurrence of clinically important metabolic-based drugdrug interactions. ${ }^{3}$

Also Phase II activity is reduced at birth, being about $50 \%-70 \%$ of the adult rate, and then increases postnatally. ${ }^{1,3}$ It includes synthetic or conjugation reactions, such as glucuronidation and sulfation. ${ }^{1}$ In particular, glucuronidation is significantly depressed during the neonatal period, while the sulfation pathway is well-developed and partially balances the low rate of glucuronidation. ${ }^{3,6}$ As a result, some drugs like acetamino- phen are excreted chiefly as sulfate conjugates in newborns while they are mainly excreted as glucuronide conjugates in adults. $^{3}$ The glucuronidation pathway reaches full capacity at about 3-4 years of life. This fact may explain the slow metabolism of some endogenous substrates such as bilirubin, and of some drugs such as morphine and chloramphenicol. ${ }^{3}$

Other organs such as the kidney may also be important for drug metabolism. ${ }^{12}$ Although the kidney is involved in the biotransformation of drugs, data on the developmental processes of renal microsomal systems are lacking., ${ }^{4,13}$

The role of the gastrointestinal microflora in drug metabolism is well known. ${ }^{14}$ At birth, infant gut colonization begins through contact with microbes from the mother. Environmental factors (hygiene, maternal or neonatal antibiotic treatments, etc) may affect the type of primary colonizing bacteria, while the neonate's diet represents the most important variable by the end of the first week of life. ${ }^{15}$

Maturation phases should be considered in developing dosage recommendations for drugs that require extensive hepatic metabolism. A particular awareness of this issue may avoid important consequences like the "gray baby syndrome," which was observed in the 1960s in newborns treated with chloramphenicol at standard pediatric doses. ${ }^{1,3}$ All the pharmacokinetic characteristics mentioned above affect the half-life value of the drug. In particular, drug halflife is four times higher in preterm infants than in adults, and is approximately two times greater in term infants compared with adults. The half-life of drugs rapidly changes in the first days of life, requiring a constant dose adjustment and monitoring of plasma concentrations of drugs with a narrow therapeutic window. ${ }^{7}$ Furthermore, it is important to consider that neonatal diseases may modify pharmacokinetic processes. In particular, the newborn undergoing intensive care, especially if preterm, frequently exhibits concurrent conditions that significantly affect the pharmacokinetics: hyperbilirubinemia, acidosis, hypothermia, hypoperfusion, increased permeability of the hematoencephalic barrier and impaired renal function. In addition, some diseases (eg, necrotizing enterocolitis) may cause specific interactions, making pharmacokinetics even more unpredictable.

Genetic factors also influence newborn ability to metabolize drugs. Therefore, important clues to neonates at risk for drug toxicity are provided by the study of pharmacogenetic predisposition. ${ }^{3}$

\section{Excretion}

The kidney is assigned to eliminate various substances including glucose, bicarbonate, and some drugs such as aminoglycosides. 
This process depends on glomerular filtration, tubular secretion, and tubular reabsorption. ${ }^{6}$ In newborn infants, renal function is significantly reduced. ${ }^{3,6}$ In particular, tubular function is less advanced than glomerular function until 6 months of life. ${ }^{6}$ Other factors contribute to the reduced renal function, namely reduced nephron length, reduced solute gradient, and diminished responsiveness to antidiuretic hormones. ${ }^{3}$

The quantity of drug filtered by the glomerulus per unit of time results from the functional ability of glomeruli, the renal blood flow integrity, and the degree of drug-protein binding. ${ }^{3}$ At birth, glomerular filtration rate is $2-4 \mathrm{~mL} / \mathrm{min}$ in term newborns; it rapidly increases to $8-20 \mathrm{~mL} / \mathrm{min}$ in the first 72 hours of life, ${ }^{3,6}$ reaching adult values at about $3-5$ months of life. ${ }^{3}$ Glomerular filtration values are lower $(0.6-0.8 \mathrm{~mL} / \mathrm{min})$ and increase more slowly in preterm infants compared to fullterm infants. ${ }^{3}$ Renal blood flow values are highly variable at birth, reaching adult values between 5 and 12 months of life. Finally, the amount of drug filtered is inversely proportional to the extent of protein binding because only the free drug is available for glomerular filtration and may be excreted in the urine. ${ }^{3}$

\section{Therapeutic drug monitoring and other tools for individualizing drug therapy}

Drug dose requirements differ markedly between newborns and older children; in addition, age-related pharmacokinetic differences and the small size of newborns can result in a high risk of medication errors, potentially leading to morbidity and mortality. Accordingly, therapeutic drug monitoring (TDM) has been considered necessary in this population, and has been used since the early 1970 s. ${ }^{16}$ The most common drugs that should be routinely monitored in the newborn are aminoglycosides and vancomycin, theophylline, caffeine, digoxin, and phenobarbital. ${ }^{17}$ TDM consists in the laboratory dosage of a specific drug at designated intervals, that later influences drug prescription. ${ }^{18}$ Initial dosing regimen for a new patient is frequently based only on past experience in a similar patient, ${ }^{19}$ so it is not always able to ensure serum drug concentrations remain within the desired range. These considerations are in favor of TDM. ${ }^{19}$ The existing relationship between dose and blood drug concentration, and between concentration and therapeutic efficacy or toxicity is the rationale for TDM use. ${ }^{18}$ Therefore, monitoring drug concentration may be useful in correcting dosing regimen and may contribute to patient safety, especially for those drugs that are characterized by concentration-dependent efficacy and toxicity rather than for those characterized by dose-dependent efficacy and toxicity. ${ }^{19}$
This measurement allows to optimize pharmacotherapy by maximizing therapeutic efficacy, while minimizing adverse events. ${ }^{16}$ Furthermore, TDM permits the individualization of drug dosage by maintaining blood drug concentration within a targeted therapeutic range. ${ }^{20}$ The use of TDM is very important for monitoring drugs with narrow therapeutic ranges such as aminoglycoside antibiotics, and difficult-to-manage medications. ${ }^{18}$ Performing TDM requires a multidisciplinary approach that involves scientists, clinicians, nurses, and pharmacists. Many indications for monitoring plasma drug concentration have been identified, including monitoring compliance, individualizing therapy during early therapy and dosage changes, diagnosing undertreatment, avoiding toxicity, monitoring and detecting drug interactions, and guiding withdrawal of therapy. There are some important points to consider in the TDM procedure to ensure reliable results. Firstly, the blood sample for measuring the drug concentration has to be obtained at the correct time after dosing in order to avoid a misinterpretation of the results. Secondly, the TDM team must be informed about the exact starting time of the drug regimen. Finally, the laboratory must ensure that the assay used is reliable and specific and that quality controls are regularly performed. Generally, plasma samples should be drawn at trough or just before the next dose. For aminoglycoside antibiotics, both peak and trough measurements are required. The peak concentration is evaluated by taking a blood sample at least 1 hour after dose administration. ${ }^{18}$ It has to be taken into account that repeated blood sampling in the newborn has been associated with neonatal anemia; therefore, laboratories that can use micromethods with samples $\leq 75 \mu \mathrm{L}$ should be preferred. Moreover, the neonatal intensive care unit (NICU) should record the amount of blood drawn for each neonate, avoiding excessive blood loss. ${ }^{17}$ Therapeutic drug measuring is only one part of TDM that needs expert and appropriate medical interpretation to ensure full clinical benefits. When interpreting plasma drug concentrations, the clinician should consider patient clinical conditions that may affect the relationship between concentration and clinical effects. In this way, the clinician can tailor the treatment to the patient's needs. ${ }^{18}$

Other modern disciplines promise the advent of “personalized medicine.” In recent years, pharmacometrics, pharmacogenomics, and pharmacometabolomics have been used as integrated approaches to optimize and individualize neonatal pharmacotherapy.

Pharmacometrics is the science of interpreting and describing pharmacology in a quantitative manner. It uses mathematical models based on biology, physiology, pharma- 
cology, and disease to quantify the interactions between drugs and patients. ${ }^{21}$ The bridging character across disciplines and the Bayesian nature of pharmacometrics have been recently emphasized. An important focus of pharmacometrics is to understand the variability in drug response. The application of a pharmacometric approach to drug development and pharmacotherapy promises to significantly improve both.

Genetic factors contribute to explain different drug responses in the newborn, influencing all pharmacokinetic and pharmacodynamic processes. This assumption has led to the development of pharmacogenetics and, more recently, of pharmacogenomics. The use of these novel tools is aimed at studying the patient genotype to optimize pharmacotherapy, thus ensuring the higher efficacy of a drug with the minimal adverse effects. However, pharmacogenetic and pharmacogenomic approaches have important limitations, first of all that of not taking into account the remarkable physiological and environmental influences on drug pharmacokinetics.

Pharmacometabolomics is a rapidly developing discipline which refers to the direct measurement of metabolites in the body fluids of an individual to predict or evaluate the metabolism of medications. ${ }^{22}$ The implementation of the pharmacometabolomic approach has the potential to greatly improve our understanding of mechanisms of drug effects, of adverse drug reactions and of the biological background of individual variations in drug response. ${ }^{3}$

\section{Medication errors}

The term "medication error" indicates any preventable event that occurs in the process of ordering, transcribing, dispensing, administering, and monitoring a drug irrespective of whether the injury occurred or potential for injury was present. According to the criteria provided by the American Society of Hospital Pharmacists, medication errors have been classified in six levels as follows: level 1, no injury; level 2 , increased clinical monitoring; level 3 , increased laboratory monitoring; level 4, required another drug or prolonged stay; level 5, permanent harm; level 6, mortality. ${ }^{23}$

Certain patient populations are at greater risk of medication errors and their negative consequences because of their inherent characteristics. It has been documented that potentially harmful errors intercepted before causing injury or those reaching the patient without any consequences are more frequent in NICU patients than in hospitalized adults. ${ }^{24}$ The newborn is more vulnerable to adverse effects of medication errors due to its small size, ${ }^{25}$ rapidly changing body surface area and weight, ${ }^{23}$ physiological immaturity, ${ }^{25}$ limited compensatory abilities, ${ }^{25}$ and inability to communicate with the provider. ${ }^{23}$ In addition, the need to calculate individualized doses and, for many drugs, the narrow therapeutic margin, increase the risk of medication errors and their adverse effects in newborns. The lack of dosage forms and concentrations appropriate for the administration to neonates, and the need for precise dose measurements and appropriate delivery systems (ie, pumps) expose the newborn to further possibilities of medication errors.

Specific characteristics of the NICU environment should be taken into consideration. The NICU is a complex adaptive system, characterized by a frequently chaotic environment with multiple unscheduled admissions of unstable newborns needing intensive care and complex medical interventions. In the NICU, medication errors are more likely to occur, potentially leading to patient injury or harm. ${ }^{26}$ About $50 \%$ of the iatrogenic complications in the NICU care have shown to be related to medication errors. ${ }^{27}$ Finally, the lack of published information on dosing, pharmacokinetics, safety, efficacy, and clinical use of drugs in the neonatal population frequently leads to the use of off-label or unlicensed drugs. Conroy et $\mathrm{al}^{28}$ found that about $55 \%$ of prescriptions in NICU contain off-label drugs and 10\% contains unlicensed drugs. In the neonatal population, unlicensed drug use was also documented to be more frequently associated with errors than licensed use. ${ }^{29}$

Medication errors have been found to be due to the combined effect of "latent failure" in the system and "active failure" by individuals. Latent failures are subtle weaknesses within an organization that normally cause no apparent consequences. Examples of latent failures are faulty information management, ineffective personnel training, stressful environment and inadequate systems of communication. When latent and active failures are combined together in a proper sequence, the system safety net is penetrated or bypassed resulting in an incident. ${ }^{30}$ Latent errors have been described by the psychologist Reason ${ }^{31}$ as the errors that are "waiting to happen." It is very important to analyze these errors because they can suggest how to alter a system in order to make it safer. ${ }^{32}$ The active failure may involve doctors, pharmacists, and nurses. Most medication errors occur because humans simply cannot perform expected tasks perfectly all of the time. ${ }^{26}$ It has been observed that dedication, training, and vigilance are not enough to prevent errors in complex systems. ${ }^{24}$ Many factors influencing the intrinsic risk of medication errors have been identified, namely intensity of workload, day shift, handovers among caregivers, lack of knowledge of the procedures, inadequate use of technology, lack of communication in the team, understaffing, inadequate training, and absence of 
awareness of errors. ${ }^{26,32,33}$ Inexperience is another risk factor for medication errors. In fact, new staff has been found to make errors more frequently because prescriptions are often written by more junior doctors that may be less familiar with drug use. ${ }^{33}$ The mental state of the person performing the action is also important. Inattention, haste, distraction, and fatigue are the main factors that have been found to influence susceptibility to error. ${ }^{32}$

The types of errors identified are different depending on the roles of NICU staff members. Doctors are mainly prone to make errors at the stage of ordering a drug. This fact is generally due to the lack of knowledge of the drug or to the lack of information about the patient. ${ }^{32}$ Ordering errors may be prescribing, transcribing, manufacturing, or compounding errors. Prescribing errors, particularly dose calculation errors, are the most frequent ones: $:^{23,34,35}$ the latter may result from miscalculation of the dose, wrong decimal point placement, incorrect expression of measurement unit, and incorrect medication administration rate. ${ }^{36}$ Overdose was the most frequent kind of medication errors while curtailed-interval was the least. ${ }^{35}$ The most frequent error in the transcription process has been found to be the absence of the administration route. ${ }^{34}$

Pharmacists make mainly dispensing errors, particularly errors in reviewing the medical prescriptions and in diluting stock solutions in order to provide extremely minute doses. $^{24}$

Nurses are mainly involved in medication preparation and administration, and are specifically trained to ensure that the right drug is given in the right dose, at the right interval, via the right route, to the right patient, with the right documentation (six rights of medication administration). Nurses are exposed to commission errors when one or more of the six rights of medication administration are violated, and to omission errors when a prescribed drug is not given to the patient. ${ }^{37}$

The most frequent administration error has been found to be the administering of medications at the wrong time. ${ }^{37-39}$ Moreover, nurses are in an important position to identify prescribing and dispensing errors, thus preventing these errors from reaching the patient. Another type of medication error that has been frequently documented in the NICU consists of patient misidentification errors. In fact, NICU patients cannot actively participate in the identification process, and they are not easily distinguishable when considering their similar appearance in the first days of life. Furthermore, wrist identification bands are often missed or removed, leading to a more difficult identification of these patients. ${ }^{40}$

The origin of medication errors in neonatology is summarized in Figure 1.

Medication error prevention should be a multidisciplinary process involving all NICU staff. ${ }^{24,41}$ There are many described interventions able to decrease NICU medication errors. Two basic strategies should be pursued: first, human behavioral factors responsible for errors have to be identified and NICU work has to be redesigned to minimize errors; second, reliable systems for preventing errors or intercepting them before they reach the patient have to be designed and employed. ${ }^{26}$ Although errors are most often due to system deficiency or failure, individual health care professionals also play a role in the prevention of medication errors. Consequently, a first intervention step to prevent this kind

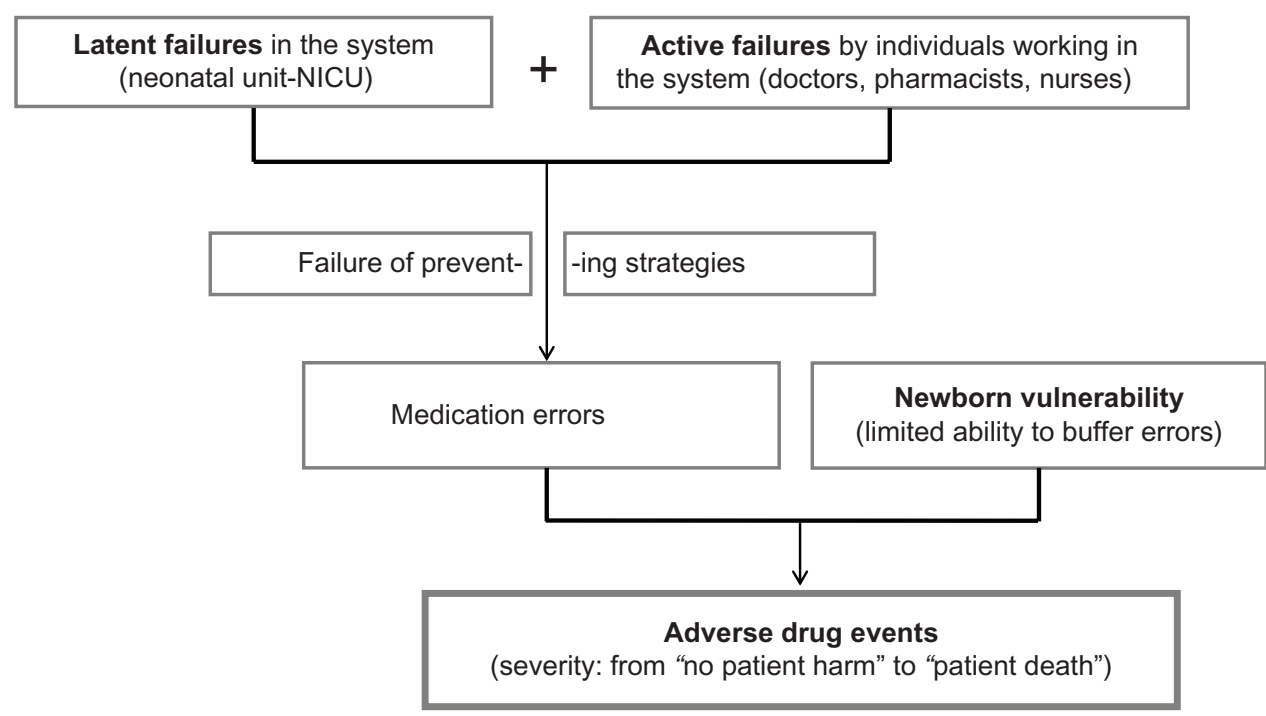

Figure I Origin of medication errors in neonatology. Abbreviation: NICU, neonatal intensive care unit. 
of error is the education and training of health care professionals. Training programs include courses to build and reinforce communication skills among health care professionals and to improve communication between caregivers and their patients, instructions and practice in performing the mathematical calculations used in patient care, and instructions about patient monitoring during therapy administration. Furthermore, many recommendations have been made to physicians in order to prevent prescription errors, emphasizing the importance of writing legible prescriptions: in this respect, of particular importance are the patient name, date of birth, current weight, allergies, any additional patient specific data appropriate to the circumstance, generic drug name, quantity to be dispensed expressed in metric units, dose, route of administration, frequency of dosing, duration of therapy, and prescriber name. ${ }^{30}$ The drug order written by physician assistants must also contain the printed name, address, telephone number, and signature of the supervising physician. Recommendations for nurses include the importance of reviewing the patient's medication administration record, verifying dosage calculations, documenting all verbal orders received from the prescriber and verifying them by repeating the orders back to him/her, verifying patient identity prior to the administration of the drug, and administering all doses at scheduled times. ${ }^{30}$ An important point is that nurses should have a detailed knowledge of the prescribed drug. This also considering that, in many countries, prescribing authority has been recently extended to nurses and other health professionals. Therefore, pharmacology education has become an important part of nurse training. Finally, clinical pharmacists could help to reduce medication errors in the NICU, considering that this category of health care professional is involved in preparing and dispensing medications based on physician prescriptions. ${ }^{30}$

Centralized reporting systems for medication errors are also recommended, since they are important tools for learning from errors. This type of preventive measure might exhibit the following main features: centralized collection; expert analysis of reports of errors, near-misses, and adverse events from health care; confidential or anonymous, nonpunitive reporting; actionable recommendations to promote patient safety. A specialty-based reporting system may be more acceptable to health care providers than a national reporting system. ${ }^{42}$ Two typologies of reporting systems have been identified: mandatory reporting systems focus on errors associated with serious injuries or death, while voluntary reporting systems focus on near-misses. ${ }^{43}$ A specialty-based, voluntary, anonymous reporting by health care professionals seems to have the higher capacity to enhance patient safety; ${ }^{42}$ moreover, a voluntary and nonpunitive approach is likely to generate valuable information on type, etiology, outcome, and preventability of incidents in the NICU. ${ }^{25}$

Available technology may contribute to preventing medication errors. Computers can provide technological support by providing information, facilitating clear and accurate communication, alerting users to potential errors, and processing data. Currently, the information technology system recommended in hospitals is the computerized physician order entry (CPOE) process, which can act as a firewall reducing the risk of medication errors. ${ }^{30}$ At present, there are no studies demonstrating the effectiveness of CPOE in reducing preventable medication errors in the NICU, but expert opinions and available data from adult and pediatric studies constitute the basis for employing CPOE in the NICU. In the full system, a physician orders a drug using CPOE and clinical decision support software; they are integrated with the pharmacy software, which controls a robot that reads the electronically delivered order and prepares a specific unit-dose of the drug to be administered to a particular patient, at a determined time and by a specific route. Finally, a barcode label containing all the above mentioned information is applied to the unit dose that is then delivered to the patient's nursing unit. The nurse takes the unit-dose package to the patient's bedside and then scans the barcode on his/her identification badge, the barcode on the patient's wristband, and the barcode on the package. The barcode scanner transmits information to another computer system that confirms the correspondence with the initial order and allows the nurse to administer the drug dose. Finally, the nurse confirms that the medication has been given so that the software enters the dose as given. ${ }^{26}$

\section{Conclusion}

The individual response to a medication in terms of efficacy and toxicity is highly variable in the neonate and this is a challenging problem in clinical practice. Predicting drug doses for neonatal population is complex as numerous physiological changes occur in early life. Both gestational and postnatal ages should be considered before administering drugs to neonates, and adaptations are necessary to maximize therapy effectiveness while limiting potential toxic effects.

A thorough knowledge of the pharmacokinetics and pharmacodynamics of a drug in neonatal and pediatric populations, which is not always available, is necessary to optimize its use. Nevertheless, neonatologists and pediatricians are currently required to use medications despite incomplete knowledge about their pharmacological properties, and the 
substantial risks inherent in the traditional approach to adjust drug dose by body size.

TDM is considered necessary for many drugs administered to the newborn infants, based on their pharmacokinetic peculiarity and the small size of these patients. However, the drug concentration should be complementary to and not a substitute for clinical judgment: it is essential to treat the individual patient and not the laboratory value.

Recent years have been characterized by a rapid development of "omic" sciences, which may be important tools to improve our understanding of mechanisms of drug effects, of adverse drug reactions and of the biology underlying individual variability in drug response. Pharmacometabolomics seems to be very promising from this point of view and, in the future, it could play an important role in designing personalized human health care. ${ }^{3}$

Another important issue concerns the major risk of medication errors in the newborn population that is mainly related to the small size and limited compensatory abilities of newborns, to the NICU complex system and to the use of off-label or unlicensed drugs.

Finally, it should be taken into account that some drugs commonly used in the neonatal period, particularly in the premature infant, may damage immature kidneys, thus increasing the risk of renal failure in adulthood.

Further clinical studies are required to improve our understanding of the behavior of drugs in newborns and infants and to provide better guidance on how to optimize drug therapy in individual patients.

\section{Disclosure}

The authors report no conflicts of interest in this work.

\section{References}

1. Skinner AV. Neonatal pharmacology. Anaesth Intensive Care Med. 2011;12(3):79-84.

2. Eberini I. Pharmacokinetics and pharmacodynamics in the newborn. Vet Res Commun. 2008;32 Suppl 1:S77-S80.

3. Fanos V, Barberini L, Antonucci R, Atzori L. Pharma-metabolomics in neonatology: is it a dream or a fact? Curr Pharm Des. 2012;18(21): 2996-3006.

4. Allegaert K, van den Anker JN, Naulaers G, de Hoon J. Determinants of drug metabolism in early neonatal life. Curr Clin Pharmacol. 2007;2(1):23-29.

5. Kaye JL. Review of paediatric gastrointestinal physiology data relevant to oral drug delivery. Int J Clin Pharm. 2011;33(1):20-24.

6. Milsap RL, Jusko WJ. Pharmacokinetics in the infant. Environ Health Perspect. 1994;102 Suppl 11:107-110.

7. Agostino R, Braguglia A, Caccamo ML, et al, editors. Farmacoterapia Neonatale. Guida Pratica Con Supporto Interattivo. 1st ed. Milano, Italy: Biomedia; 2009. Italian.

8. Goutières F, Aicardi J. Accidental percutaneous hexachlorophane intoxication in children. Br Med J. 1977;2(6088):663-665.
9. Cheymol G. Effects of obesity on pharmacokinetics implications for drug therapy. Clin Pharmacokinet. 2000;39(3):215-231.

10. Blake MJ, Castro L, Leeder JS, Kearns GL. Ontogeny of drug metabolizing enzymes in the neonate. Semin Fetal Neonat Med. 2005;10:123-138.

11. Krishna DR, Klotz U. Extrahepatic metabolism of drugs in humans. Clin Pharmacokinet. 1994;26:144-160.

12. Alcorn J, McNamara PJ. Ontogeny of hepatic and renal systemic clearance pathways in infants: Part I. Clin Pharmacokinet. 2002;41: 959-998.

13. Ward RM. Drug disposition in the late preterm ("near-term") newborn. Semin Perinatol. 2006;30(1):48-51.

14. Shamat MA. The role of the gastrointestinal microflora in the metabolism of drugs. Int J Pharm. 1993;97:1-13.

15. Langhendries JP, Paquay T, Hannon M, Darimont J. Intestinal flora in the neonate: impact on morbidity and therapeutic perspectives. Arch Pediatr. 1998;5(6):644-653. French.

16. Touw DJ, Neef C, Thomson AH, Vinks A. Cost-effectiveness of therapeutic drug monitoring. Ther Drug Monit. 2005;27:10-17.

17. Koren G. Therapeutic drug monitoring principles in the neonate. National Academy of Clinical Biochemistry. Clin Chem. 1997;43(1): 222-227.

18. Kang J, Lee M. Overview of therapeutic drug monitoring. Korean J Intern Med. 2009;24:1-10.

19. Touw DJ, Westerman EM, Sprij AJ. Therapeutic drug monitoring of aminoglycosides in neonates. Clin Pharmacokinet. 2009; 48(2):71-88.

20. Birkett DJ. Pharmacokinetics made easy: therapeutic drug monitoring. Austr Prescr. 1997;20:9-11.

21. Zhang L, Pfister M, Meibohm B. Concepts and challenges in quantitative pharmacology and model-based drug development. AAPS J. 2008;10(4):552-559.

22. Kaddurah-Daouk R, Kristal BS, Weinshilboum RM. Metabolomics: a global biochemical approach to drug response and disease. Annu Rev Pharmacol Toxicol. 2008;48:653-683.

23. Jain S, Basu S, Parmar VR. Medication errors in neonates admitted in intensive care unit and emergency department. Indian J Med Sci. 2009;63(4):145-151.

24. Gray JE, Goldmann DA. Medication errors in the neonatal intensive care unit: special patients, unique issues. Arch Dis Child Fetal Neonatal Ed. 2004;89:F472-F473.

25. Snijders C, van Lingen RA, Molendijk A, Fetter WPF. Incidents and errors in neonatal intensive care: a review of the literature. Arch Dis Child Fetal Neonatal Ed. 2007;92:391-398.

26. Morriss FH. Adverse medical events in the NICU: epidemiology and prevention. Neo Reviews. 2008;9:e8-e23.

27. Sekar KC. Iatrogenic complications in the neonatal intensive care unit. J Perinatol. 2010;30 Suppl:S51-S56.

28. Conroy S, McIntyre J, Choonara I. Unlicensed and off label drug use in neonates. Arch Dis Child Fetal Neonatal Ed. 1999;80:F142-F145.

29. Conroy S. Association between licence status and medication errors. Arch Dis Child. 2011;96(3):305-306.

30. Levine SR, Cohen MR, Blanchard NR, et al. Guidelines for preventing medication errors in pediatrics. J Pediatr Pharmacol Ther. 2001;6:426-442.

31. Reason JT. Human Error. Cambridge, UK: Cambridge University Press; 1990.

32. McDowell SE, Ferner HS, Ferner RE. The pathophysiology of medication errors: how and where they arise. Br J Clin Pharmacol. 2009;67(6):605-613.

33. Simpson JH, Lynch R, Grant J, Alroomi L. Reducing medication errors in the neonatal intensive care unit. Arch Dis Child Fetal Neonatal Ed. 2004;89:F480-F482.

34. Campino Villegas A, Lopez Herrera MC, Garcia Franco M, Lopez de Heredia Goya I, Valls i Soler A. Medication prescription and transcription errors in a neonatal unit. An Pediatr (Barc). 2006;64(4):330-335. Spanish. 
35. Kazemi A, Ellenius J, Pourasghar F, et al. The effect of Computerized Physician Order Entry and decision support system on medication errors in the neonatal ward: experiences from an Iranian teaching hospital. J Med Syst. 2011;35(1):25-37.

36. Lesar TS, Lomaestro BM, Pohl H. Medication-prescribing errors in a teaching hospital: a 9-year experience. Arch Intern Med. 1997;157: 1569-1576.

37. Raja Lope RJ, Boo NY, Rohana J, Cheah FC. A quality assurance study on the administration of medication by nurses in a neonatal intensive care unit. Singapore Med J. 2009;50(1):68-72.

38. Wilson DG, McArtney RG, Newcombe RG, et al. Medication errors in paediatric practice: insights from a continuous quality improvement approach. Eur J Pediatr. 1998;157(9):769-774.

39. Barker KN, Flynn EA, Pepper GA, Bates DW, Mikeal RL. Medication errors observed in 36 health care facilities. Arch Intern Med. 2002; 162(16):1897-1903.
40. Gray JE, Suresh G, Ursprung R, et al. Patient misidentification in the neonatal intensive care unit: quantification of risk. Pediatrics. 2006; 117(1):e43-e47.

41. Dabliz R, Levine S. Medication safety in neonates. Am J Perinatol. 2012;29(1):49-56.

42. Suresh G, Horbar JD, Plsek P, et al. Voluntary anonymous reporting of medical errors for neonatal intensive care. Pediatrics. 2004;113(6): $1609-1618$

43. Ligi I, Arnaud F, Jouve E, Tardieu S, Sambuc R, Simeoni U. Iatrogenic events in admitted neonates: a prospective cohort study. Lancet. 2008; 371:404-410.

\section{Publish your work in this journal}

Research and Reports in Neonatology is an international, peer-reviewed, open access journal publishing original research, reports, editorials, reviews and commentaries on neonatal health. The manuscript management system is completely online and includes a very quick and fair peer-review system. Visit http://www.dovepress.com/testimonials.php to read real quotes from published authors. 